\title{
Tolerance of Dosed Physical Loads in the Rehabilitation of Patients With a Recent Experience of Myocardial Infarction or Unstable Angina
}

Mu Chenguang ( $\nabla$ muchenguang79@gmail.com )

Postgraduate of the National University of Physical Education and Sport of Ukraine

Kushakova Nadezhda Ivanovna

Donetsk National Medical University

Bobyrev Vladimir Evgenievich

Donbass State Pedagogical University

\section{Research Article}

Keywords: therapeutic exercise, physical activity, myocardial infarction, heart rate, blood pressure, functional state

Posted Date: June 21st, 2021

DOI: https://doi.org/10.21203/rs.3.rs-612621/v1

License: (9) (1) This work is licensed under a Creative Commons Attribution 4.0 International License. Read Full License

Version of Record: A version of this preprint was published at Journal of Cardiology and Cardiovascular Research on January 2nd, 2022. See the published version at https://doi.org/10.37191/Mapsci-JCCR-3(1)-048. 


\section{Abstract}

Objective: The study of reaction to dosed physical load of patient with recent experience of myocardial infarction or unstable angina to assess objectively physical condition and performance, to appoint an adequate individual program of physical rehabilitation and to optimize the effectiveness of the sanatorium stage of rehabilitation of this category of patients.

Design: Study and observational analysis.

Parameter: The subsidiary «Sanatorium-resort rehabilitation center «Slaviansk resort», the sanatorium «Yubileiny», Ukraine.

Participants: Patients with recent experience of myocardial infarction or unstable angina who arrived at the sanatorium stage of rehabilitation from cardiological hospitals of Donetsk and Lugansk regions (Ukraine).

Intervention: Non-invasive treatment (i.e. therapeutic exercise)

Results: 3530 patients who arrived at the sanatorium stage of rehabilitation from cardiological hospitals of Donetsk and Lugansk regions (Ukraine) on $29,4 \pm 2,4$ day of illness were examined. Men accounted for $64 \%$, women $-36 \%$. The average age of the patients was $54,4 \pm 1,8$ years old. 1127 people (31,93\%) had a Q-positive myocardial infarction (MI), 388 people (10,99\%) - a Q-negative, 2015 people $(57,08 \%)$ - an unstable angina.

When studying the tolerance to dosed physical activity, threshold or inadequate reactions were observed in 144 patients (12.78\%) who had Q-positive myocardial infarction, 13 patients (3.35\%) who had Q-negative myocardial infarction and 89 patients (4.42\%) who had unstable angina. Threshold or inadequate responses in patients with recent experience of Q-positive myocardial infarction were recorded 3-4 times more often. The electrocardiographic examination revealed most often changes in the terminal part of the ventricular complex in the form of inversion or reversal of $T$ waves, to a greater extent in patients who had Q-positive myocardial infarction.

Conclusions: It was found in the course of study that patients with recent experience of Q-positive myocardial infarction master less physical activity at the sanatorium stage of rehabilitation than patients of other groups.

Our study has shown that an insufficient level of physical activity does not give an optimal effect in increasing the functional reserves of the cardiovascular system and the body as a whole, and insufficient control of an increase in the level of physical activity is fraught with negative consequences.

Careful selection of optimal physical activity for this category of patients allowed to prescribe adequate physical activity to patients and avoid possible complications.

\section{Introduction}

\section{Relevance.}

The main task of the sanatorium-resort stage of rehabilitation of patients who have suffered acute myocardial infarction or unstable angina is to expand motor activity, which is achieved through the correct construction of the motor regime, taking into account the functional state of the patient. All measures of the sanatorium-resort stage of rehabilitation are carried out to patients differentially, depending on the condition, features of the clinical course of the disease, the presence of pathological syndromes and concomitant diseases. 1, 2 During physical rehabilitation, various types of physical training are used: therapeutic exercise both in a gentle and training mode, with increasing load. Dosed walking remains the most accessible and common training method.

Patients with uncomplicated myocardial infarction should be encouraged to increase their physical activity. It is necessary to expand the mode of physical activity of patients with a high risk of developing cardiovascular disease under the guidance of therapeutic exercise specialists and participate in controlled physical exercise programs. 
To optimize the effectiveness of treatment at the sanatorium stage of rehabilitation of patients with recent experience of acute myocardial infarction or unstable angina, it is of great importance to determine individual tolerance to physical activity and methods of objective control of their tolerance. $3,4,12,13,14,15$

The most common forms of therapeutic exercise at the sanatorium stage of rehabilitation are dosed (therapeutic) walking, movement therapy, elements of sedentary and sports games, as well as walking and training walking up the stairs. Special attention is paid to medical walking, since this type of activity leads to systemic adaptation of the body to physical activity, namely: the performance of the heart muscle improves, the skeletal muscles are strengthened and the blood supply to organs and systems is improved due to the activation of the respiratory system. $5,6,7,8,16,17,18,19$

The aim of the study. is to research the response to dosed physical activity of patients with recent experience of myocardial infarction or unstable angina to assess objectively physical condition and performance.

To achieve this goal, we solved the following tasks:

1. To assess the level of physical activity in patients with coronary heart disease at the sanatorium stage of rehabilitation.

2. To determine the criteria for the threshold load of patients with acute myocardial infarction or unstable angina at the sanatorium stage of rehabilitation.

\section{Methods}

A feature of physical training in patients with ischemic heart disease at the present stage is a well-founded tendency to an increase in the level of general physical activity. A prerequisite for the correct use of various forms of therapeutic exercise is the correspondence of the volume and intensity of physical activity to the clinical manifestations of the disease and the functional capabilities of the organism. 3, 4, 7, 10, 12, 14 An insufficiently controlled increase in the level of physical activity is fraught with negative consequences, and an insufficient level of physical activity does not give an optimal effect in increasing the functional reserves of the cardiovascular system and the body as a whole. Therefore, stress tests are taking an increasing place in the complex of modern cardiological research. In recent years, such physical exercise tests such as bicycle ergometry have been used less frequently due to possible complications. Authors of a number of publications propose to include the presence of stenosis of the left coronary artery $\mathbf{5 0 \%}$ or more in the list of contraindications for conducting a bicycle ergometric study. Coronary angiographic examination of this category of patients who arrived at the sanatorium stage of rehabilitation was carried out at the hospital stage to a few patients. In the conditions of the sanatorium, the determination of tolerance to physical activity is carried out mainly to various forms of therapeutic exercise, taking into account the level of activity (LA) and functional state, which gives a more objective assessment of the functional state of the cardiovascular system of each patient. 2, 5, 9, $11,13,20,21$

The data of this study are based on 5-year experience of the cardiology department of rehabilitation of patients with recent experience of myocardial infarction and unstable angina.

We examined 3530 patients who arrived at the sanatorium stage of rehabilitation from cardiological hospitals of Donetsk and Lugansk regions (Ukraine), on the $29.4 \pm 2.4$ day of illness. Men accounted for $64 \%$, women $-36 \%$. The average age of the patients was $54.4 \pm 1.8$ years old. 1127 people (31.93\%) suffered Q-positive myocardial infarction, 388 people (10.99\%) - Qnegative, 2015 people (57.08\%) - unstable angina.

At the time of admission to the sanatorium, 338 patients (34.43\%) with Q-positive myocardial infarction mastered the $4^{\text {th }}$ stage of activity at the hospital stage (walking 500 meters or more at a speed of 60-80 steps per minute, climbing 22 or more steps with a speed of 1 step per 1 second and a complex of medical gymnastics standing and sitting), 626 people (55.55\%) mastered the $3^{\text {rd }}$ step (walking less than 500 meters and at a speed of up to 60 steps per 1 minute and a complex of medical gymnastics lying or sitting), 113 patients $(10.02 \%)$ of this group were admitted unadapted (they walked only within the ward and performed therapeutic exercises while lying in bed). 330 people (85.05\%) mastered the $4^{\text {th }}$ stage of activity, and 58 people 
(14.95\%) mastered the $3^{\text {rd }}$ stage in the group of patients who had Q-negative myocardial infarction. Among 2015 patients who had unstable angina, $100 \%$ were admitted to the sanatorium.

\section{Statistical Analysis}

The described statistics were used to study the level of physical activity at the sanatorium stage of rehabilitation in patients with recent experience of Q-positive myocardial infarction, Q-negative myocardial infarction, or unstable angina.

Mean values and standard deviations were used to study the level of physical activity at the sanatorium stage of rehabilitation in patients with Q-positive myocardial infarction, Q-negative myocardial infarction, or unstable angina.

\section{Results}

As can be seen from the Table 1, there is the smallest increase in the level of physical activity of the patients with coronary heart disease and a group of patients who have had Q-positive myocardial infarction at the sanatorium stage of rehabilitation. The program of physical rehabilitation at the sanatorium stage is mastered in almost the same volume by patients who have had Qnegative myocardial infarction or unstable angina.

When analyzing the criteria for inadequate exercise tolerance, it turned out that most often we observed changes in the amplitude of positive or negative $T$ waves on the electrocardiogram in the form of deepening or increasing the height of the "coronary" T waves. The generally accepted criteria of the threshold load were taken into account: an attack of angina pectoris, inadequate fluctuations in blood pressure and heart rate, displacement or elevation of the ST segment from the isoline, a change in the end part of the ventricular complex of the electrocardiogram (deepening of negative T waves or increasing the amplitude of positive T waves, reversal or inversion of T waves) , rhythm or conduction disturbances, general weakness, sharp pallor of the face or increased cyanosis, the patient's refusal to continue the load.

The results of tests with dosed physical activity were assessed by us as "adequate", "inadequate" or "threshold". When studying the tolerance to dosed physical activity, we observed threshold or inadequate responses in 144 patients (12.78\%) who had Qpositive myocardial infarction, in 13 patients (3.35\%) who had Q-negative myocardial infarction and 89 patients (4.42\%) who had unstable angina pectoris. As can be seen, threshold or inadequate reactions in patients of the first group were recorded 3-4 times more often.

The criteria for inadequate tolerance of dosed physical activity of patients with coronary heart disease at the sanatorium stage of rehabilitation are presented in the Table 2. Most often, electrocardiographic examination revealed changes in the terminal part of the ventricular complex in the form of inversion or reversal of T waves, to a greater extent in patients who have had Qpositive myocardial infarction.

\section{Discussion}

Currently, there is an active development of rehabilitation programs for patients with coronary heart disease and tendencies towards their individualization and the inclusion of new complex techniques, which expands the effective use of new possibilities of restorative medicine. Only the active participation of patients with coronary heart disease in cardiac rehabilitation increases the long-term adherence of these patients to subsequent lifestyle changes. In this regard, patients of this category need to learn independent therapeutic exercise and methods of self-control over the tolerance of physical training. Pulse count was performed and the training and threshold limits both in the process of the sanatorium stage of rehabilitation and upon discharge to the outpatient stage of rehabilitation were determined for each patient, which was reflected in the abstract of medical record to ensure the continuity of the rehabilitation process.

Physical rehabilitation using physical training programs is of primary importance in the system of cardiac rehabilitation, as it allows the patient to restore physical performance, return to the usual household loads and social activity. This allows patients who have suffered acute myocardial infarction or unstable angina to maintain their quality of life. 


\section{Limitations}

Exercise tests are considered a mandatory research method in patients with coronary heart disease. However, there are the following contraindications for carrying out stress tests:

1. Severe circulatory insufficiency.

2. Frequent attacks of angina pectoris

3. Dangerous rhythm disturbances (high-grade arrhythmias).

4. Severe aortic stenosis.

5. Fresh embolism of vessels of a large or small circle of blood circulation.

6. Active or fresh thrombophlebitis.

7. Acute infectious diseases. 2, 13, 20, 21

Relative contraindications are:

1. "Unverified", often recurring supraventricular rhythm disturbances, group extrasystoles.

2. Severe arterial hypertension (blood pressure above 160 / 100-110 mm Hg. Art.).

3. Heart aneurysm with initial signs of circulatory insufficiency.

4. Moderate aortic stenosis.

5. Diabetes mellitus, thyrotoxicosis, myxedema (subcompensated).

Conditions requiring special precautions:

1. Conduction disorders: complete atrioventricular block, left bundle branch block, Wolff-Parkinson-White syndrome.

2. Electric cardiac pacemaker.

3. Controlled rhythm disturbances.

4. Electrolyte imbalance.

5. Pre-intake of digitalis drugs, beta-blockers, diuretics (they change the reaction of the cardiovascular system during exercise significantly).

6. Severe arterial hypertension with diastolic pressure above $100 \mathrm{~mm} \mathrm{Hg}$. Art., retinopathy of the 2nd stage.

7. Severe anemia.

8. Acute obesity.

9. Diseases of the kidneys, liver and other manifestations of metabolic insufficiency.

10. Psychoneurotic deviations.

11. Diseases of the organs of movement and support, limiting active mobility.

\section{Conclusion}

Thus, in the course of our study, it was found that patients with recent experience of Q-positive myocardial infarction master less physical activity at the sanatorium stage of rehabilitation than patients of other groups. The highest frequency of threshold reactions in response to the dosed load at the sanatorium stage of rehabilitation is observed in patients who have had Qpositive myocardial infarction. This group of patients requires a more careful selection at the sanatorium stage of rehabilitation and a more thorough study of tolerance to various kinds of functional loads, such as: climbing stairs, breathing exercises and complexes of physiotherapy exercises. 
Coronary artery disease in old age is characterized by high prevalence and clinical diversity. Women have less severe forms than men. Epidemiological criteria for coronary heart disease on the electrocardiogram can be considered both diagnostic and criteria for stratification of patients according to the degree of risk of mortality from cardiovascular diseases and all causes.

A prerequisite for the correct use of various forms of physiotherapy exercises is the correspondence of the volume and intensity of physical activity to the clinical manifestations of the disease and the functional capabilities of the body. An increase in physical activity is fraught with negative consequences, as our study has shown, and its insufficient level does not give an optimal effect in increasing the functional reserves of the cardiovascular system and the body as a whole. Our study of tolerance to various kinds of physical activity in this category of patients made it possible to prescribe adequate physical activity to patients and avoid possible complications. Taking into account that the effectiveness of the sanatorium stage of rehabilitation was lower in patients who had Q-positive myocardial infarction, than in patients of other groups, it should be noted that, thanks to a thorough study of exercise tolerance, there were no cases of deterioration by sanatorium stage of rehabilitation in this group of patients. All patients included in this research program were discharged from the sanatorium for the polyclinic stage of rehabilitation with improvement. Upon discharge, each patient received recommendations for further expansion of the motor regimen, which is reflected in the discharge from the medical history sheet.

PERSPECTIVE

It was noticed that most of the studied patients of these groups showed different reactions to weather conditions. Therefore, meteorological dependence in patients with coronary heart disease should be further investigated, systematized and taken into account when developing rehabilitation programs.

For the stable consolidation of the results of the sanatorium stage of rehabilitation of patients upon discharge, it is necessary to develop rehabilitation programs for each patient, taking into account physical activity and daily household loads.

Additional experimental studies of the effect of various types of balneological treatment of patients with coronary heart disease are needed. And also the use of natural healing factors of the Slaviansk resort (brine, mineral drinking water, mud therapy and medicinal peloid preparations).

Pharmacophysiotherapy also requires a deeper study - an effective combination of various physical factors with the use of medications for this category of patients.

A separate task is also to optimize the sanatorium stage of rehabilitation of patients who have had acute myocardial infarction against the background of diseases of the musculoskeletal system, which affects the development of physical training negatively. This category of patients needs to look for such types of physical activity that can be performed by patients and be an alternative to dosed walking. It is also necessary to pay attention to psychotherapy, in particular, to mediate physical exercise and develop a high commitment to physical training.

\section{Declarations}

\section{Ethics approval and consent to participate}

Consent for publication:

This study and the consent form were approved by the Donbass State Pedagogical University. Signed written informed consent was obtained from all subjects.

Availability of data and material:

The datasets used and analyzed during the current study are available from the corresponding author on reasonable request.

Competing interests:

Not applicable. 


\section{Funding:}

Not applicable.

\section{Authors' contributions:}

None.

The study adheres to STROBE guidelines and a completed STROBE checklist has been submitted separately.

Acknowledgements: The authors would like to thank the chief physician of the sanatorium "Yubileiny», the subsidiary «Sanatorium-resort rehabilitation center «Slaviansk resort», Ph.D., Medicine, Physician of Superior Merit, Yakovlenko Aleksey Viktorovich.

\section{Institutions where data was collected:}

Subsidiary «Sanatorium-resort rehabilitation center «Slaviansk resort», sanatorium «Yubileiny»

\section{Conflicts of interest / funding:}

There are no significant financial or commercial conflicts of interest.

The authors did not receive funding for this study.

The work was carried out in accordance with generally accepted bioethical norms, in compliance with the relevant laws of Ukraine.

The authors thank all patients who allowed their data to be used anonymously for study.

\section{References}

1. Basics of physical rehabilitation / G.P. Mahliovana - Lviv: Liga-Pres - 2006. - 148 p.

2. Sanatorium stage of rehabilitation of patients with ischemic heart disease / V.A. Bobrov, I.K. Sledzevskaya, M.V. Loboda and etc.- K.: Zdorovia, 1995. - 112 p.

3. E.I. Sorokina. Physical methods of treatments in cardiology. - M.: Meditsina, 1989. - 384 p.

4. V.P. Zaitsev. Physical rehabilitation of patients with myocardial infarction. - Kharkov: Kharkiv State Academy of Physical Culture, 1995. - $147 \mathrm{p}$.

5. Therapeutic physical training in sanatoriums. Edited by L.I. Fisenko. - Kyiv. - 2005. - 402 p.

6. Л.F. Nikolaeva, D.M. Aronov. Rehabilitation of patients with coronary heart disease. - M.: Meditsina, 1988. -288 p.

7. New aspects of treatment and rehabilitation of patients with myocardial infarction. - K.: Ukrainian Research Institute of Cardiology named after Academician M.D. Strazhesko, 1997. - 34 p.

8. O.F. Misyuta, V.N. Shestakov, I.A. Zobenko, A.V. Karpukhin. Sanatorium cardiological rehabilitation. CPB.: Spec. lit, 2013. $192 \mathrm{p}$.

9. M.L. Palok, D.H. Schmidt. Heart disease and rehabilitation. Kiew: Olympic literature, 2000. - 402 p.

10. Diagnostics and treatment of patients with acute myocardial infarction with ST-segment elevation on ECG. Russian recommendations. 2014. 149 p.

11. V.S. Sukhan. Therapeutic exercise in diseases of the cardiovascular system: methodical recommendations/V.S. Sukhan, L.V. Dychka, O.S. Blaga. - Uzhhorod, 2014. - 62 p.

12. S.S. Volkova. Theoretical aspect of physical rehabilitation of patients with myocardial infarction. Physical culture, sports and health of the nation: collection of scientific works / Editor in Chief V.M. Kostyukevich. - Khortytsia - 2017. - 258 p. 
13. Rehabilitation and secondary prevention in patients with acute ST-segment elevation myocardial infarction. Methodical recommendations. Moscow - 2014 - p. 94.

14. Cardiovascular diseases. Classification, standards of diagnosis and treatment / All-Ukrainian Association of Cardiologists; Edited by V.M. Kovalenko [etc.]. - the $4^{\text {th }}$ edition, revised and supplemented - Kyiv: Morion, 2020. - $240 \mathrm{p}$.

15. Physical rehabilitation: Textbook for students of higher educational institutions studying according to the State educational standard 022500 "Physical culture for persons with health deviations" (Adaptive physical education) / Under general editorship of S.N. Popov. The $3^{\text {rd }}$ edition - Rostov n/D: Feniks, 2005. - 608 p.

16. I.M. Grigus, Л.B. Brega. Physical therapy in cardiology. National University of Water Management and Environmental Sciences, Rivne. - 2018.

17. Therapeutic exercise -V.A. Epifanov - Textbook - M.: GEOTAR - Media, 2006. - 586 p.

18. V.M. Mukhin. Physical rehabilitation / V.M. Mukhin. - K.: Olympic literature, 2005. - 436 p.

19. Acute myocardial infarction with ST segment elevation on the electrocardiogram: rehabilitation and secondary prevention. Russian Journal of Cardiology No. 1 (117) - 2015.

20. Rehabilitation of patients after myocardial infarction. Recommendations for diagnosis and treatment. Under the editorship of F.I. Belyalov. Irkutsk, 2015. - 24 p.

21. S.G. Sudzhaeva, T.S. Gubich, N.A. Kazaeva. Rehabilitation of patients of cardiological and cardiac surgery profile (cardiac rehabilitation). National guidelines. Minsk. Prof. Edit. - 2010 - 158-285 p.

\section{Tables}

Table 1. The level of physical activity in patients with ischemic heart disease at the sanatorium stage of rehabilitation

$$
\text { Upon admission to the sanatorium Upon discharge from the sanatorium }
$$

Not adapted

Diagnosis $\quad 3^{\text {rd }} \mathrm{AL} \quad 4^{\text {th }} \mathrm{AL} \quad 4^{\text {th }} \mathrm{AL} \quad 5^{\text {th }} \mathrm{AL} \quad 6^{\text {th }} \mathrm{AL} \quad 7^{\text {th }} \mathrm{AL}$

$\begin{array}{lllllllllllllll}\mathrm{n} & \% & \mathrm{n} & \% & \mathrm{n} & \% & \mathrm{n} & \% & \mathrm{n} & \% & \mathrm{n} & \% & \mathrm{n} & \%\end{array}$

\begin{tabular}{lllllllllllllll}
$\begin{array}{l}\text { Q-positive } \\
\text { MI }\end{array}$ & 626 & 55,55 & 388 & 34,43 & 113 & 10,02 & 337 & 29,9 & 554 & 49,16 & 225 & 19.96 & 11 & 0,98 \\
\hline $\begin{array}{l}\text { Q-negative } \\
\text { MI }\end{array}$ & 58 & 14,95 & 330 & 85,05 & - & - & 21 & 5,41 & 165 & 42,53 & 176 & 45,36 & 26 & 6,7 \\
\hline $\begin{array}{l}\text { Unstable } \\
\begin{array}{l}\text { angina with } \\
\text { stabilization } \\
\text { in 1,2 or } \\
\text { functional } \\
\text { class }\end{array}\end{array}$ \\
\end{tabular}
$\mathrm{AL}$ - activity level
$\mathrm{Ml}$ - myocardial infarction

Table 2. Criteria for the threshold load of patients with ischemic heart disease 


\begin{tabular}{|c|c|c|c|c|c|c|}
\hline \multirow[t]{2}{*}{ Inadequacy criteria } & \multicolumn{2}{|c|}{ Q-positive Ml } & \multicolumn{2}{|c|}{ Q-negative Ml } & \multicolumn{2}{|c|}{ Unstable angina } \\
\hline & $\mathrm{n}$ & $\%$ & $\mathrm{n}$ & $\%$ & $\mathrm{n}$ & $\%$ \\
\hline Pain syndrome & - & - & 1 & 0,26 & 2 & 0,1 \\
\hline ST segment elevation above the isoline & 5 & 0,44 & - & - & - & - \\
\hline ST segment depression & 19 & 1,69 & 3 & 0,77 & 13 & 0,65 \\
\hline T wave reversal & 75 & 6,65 & 25 & 6,44 & 22 & 1,09 \\
\hline T wave inversion & 50 & 4,44 & 26 & 6,7 & 33 & 1,64 \\
\hline Rhythm disturbances & 2 & 0,18 & 1 & 0,26 & 6 & 0,3 \\
\hline Inappropriate heart rate change & 2 & 0,18 & 1 & 0,26 & 1 & 0,05 \\
\hline Inappropriate changes in blood pressure & 1 & 0,09 & 2 & 0,52 & 3 & 0,15 \\
\hline
\end{tabular}

